\title{
Influence de l'agitation du lait cru sur les résultats de dénombrement de sa flore totale à l'aide d'une anse calibrée
}

\author{
par \\ J. RICHARD*
}

\section{INTRODUCTION}

Les micro-organismes présents dans le lait cru sont souvent réunis en amas comprenant un nombre plus ou moins élevé de cellules. Ces amas résultent, soit de la division sans dispersion des micro-organismes dans le lait (exemple des staphylocoques et des streptocoques), soit de l'agglutination de certains micro-organismes par des immunoglobulines (agglutinines) présentes dans le lait cru, comme l'ont montré Auclair et Portman [4], Emmons et al. [10], Randolph et Gould [21], Stadhouders et Hup [24], pour les streptocoques lactiques. Ces immunoglobulines fixent les amas microbiens sur les globules gras qu'elles ont également agglutinés [7].

Les micro-organismes du lait cru peuvent également se trouver en grands nombres sur (ou dans) des particules solides introduites dans le lait au cours de la traite (particules de bouse sèche, fragments de "pierre " de lait...) et qui ne se désagrègent pas spontanément dans le lait.

Ces amas microbiens sont plus ou moins dispersés au cours de l'agitation du lait précédant la numération et au cours de l'agitation des dilutions de lait lors de cette opération. Il en résulte que l'on constate souvent des écarts significatifs, parfois importants, dans les dénombrements effectués sur les mêmes échantillons, par des personnes appartenant au même laboratoire ou à des laboratoires différents $[9,14]$. C'est probablement pour cette raison que la technique de dénombrement simplifiée à l'aide d'une anse calibrée donne parfois des résultats différents de la méthode classique par dilutions, seulement avec certaines catégories de lait $[27,31,32,12$ a].

\footnotetext{
* Laboratoire de Microbiologie Laitière et de Génie Alimentaire, Institut National de la Recherche Agronomique - F 78350 Jouy-en-Josas (France).
} 
Par ailleurs, du fait de la présence dans le lait peu agité d'agrégats de matière grasse plus ou moins gros, ou inversement de mousse et de grains de beurre, lorsque le lait est agité trop vigoureusement, on peut s'attendre à ce que les résultats d'une méthode de dénombrement microbien qui repose sur l'inoculation des boîtes de Petri avec un très faible volume de lait $(0,01$ ou $0,001 \mathrm{ml})$ prélevé à la surface de l'échantillon, dépendent en partie de la technique d'agitation du lait.

En dépit de l'intérêt du dénombrement simplifié à l'aide d'une anse calibrée, et malgré les critiques dont il a parfois été l'objet [27, $31,32,12$ a], peu d'études du mode d'agitation du lait cru recommandé pour cette méthode on été publiées. Leesment et Nickels [19] ont montré, avec du lait écrémé ensemencé avec une souche de Leuconostoc citrovorum formant de longues chaînes de cellules, qu'une agitation mécanique d'au moins $1 \mathrm{~min}$ était nécessaire pour que le dénombrement à l'aide d'une anse calibrée donne des résultats comparables à celui faisant intervenir des dilutions. Ces résultats n'ont cependant pas été vérifiés dans les conditions pratiques (lait entier cru à différents niveaux de contamination microbienne). Les «Standard Methods " [3] pour leur part, recommandent pour le dénombrement avec dilutions et pour les méthodes simplifiées ( Oval tube method » et "Plate loop method »), des agitations apparemment différentes et qui ne semblent pas avoir fait l'objet d'une évaluation.

Nous avons entrepris, au laboratoire, différentes études comparatives du dénombrement simplifié à l'aide d'une anse calibrée proposée par Thompson et al. [29] et du dénombrement de la flore totale du lait cru mettant en œuvre des dilutions décimales. Le présent article est consacré principalement à l'influence de l'agitation du lait cru sur la précision* et la répétabilité* du dénombrement simplifié.

\section{METHODES}

\section{Dénombrements microbiens à partir de lait cru}

a) MÉthode SIMPlifiée de THOMPSON (MST)

\section{- Agitation du lait}

Des échantillons de lait réfrigéré (mélange de deux ou quatre traites) ont été prélevés dans une quinzaine de fermes utilisant diverses techniques de nettoyage de la vaisselle laitière. Ces échantillons étaient conservés à $0^{\circ} \mathrm{C}$ jusqu'au moment de l'analyse ( 3 à $4 \mathrm{~h}$ plus tard).

\footnotetext{
* Pour la signification de ces termes voir références [1] et [12 b].
} 
Après répartition des échantillons en sous-échantillons, le lait a subi les modes d'agitation suivants :

AS : agitation "standard » consistant à secouer l'échantillon à la main pendant $10 \mathrm{~s}$, le flacon effectuant 25 allers et retours d'une amplitude de $30 \mathrm{~cm}$ environ [3],

V: agitation "standard » suivie d'une agitation par vibrations à l'aide d'un appareil Vortex* réglé au maximum. Pour faciliter cette agitation, le lait était placé dans un tube de $18 \times 180$, puis transvasé sans la mousse dans une capsule pour le prélèvement à l'anse.

Des durées d'agitation par Vortex de 15 s, 30 s, 1 et 2 min ont été expérimentées,

US : agitation par ultrasons produits par un générateur Metason (Struers) dans une cuve de 1,6 l remplie au 1/5 avec de l'eau. Les ondes ultrasoniques utilisées avaient une fréquence de $80 \mathrm{kHz}$ et une intensité acoustique moyenne de $0,4 \mathrm{~W}$ par $\mathrm{cm}^{2}$. Le lait (environ $5 \mathrm{ml}$ ) était mis dans un flacon de type pilulier placé au centre de la cuve. Différentes combinaisons de durées et de températures ont été essayées,

UT : agitation pendant $30 \mathrm{~s}$ à l'aide d'une turbine tournant à $20000 \mathrm{t} / \mathrm{min}$ (appareil Ultra-Turrax type 18/2). La mousse produite était éliminée par transvasement avant le prélèvement à l'anse.

\section{- Inoculation des boites de Petri}

La méthode décrite par Thompson et al [29] a été suivie à l'exception près que le prélèvement du lait avec la boucle calibrée a été mécanisé : la canule et la boucle calibrée ont été placées verticalement sur un support fixe, l'échantillon sur un support se déplaçant verticalement au moyen d'un dispositif à crémaillère entraîné par un moteur électrique. Ce dispositif permettait de régler à la main la profondeur de pénétration de la boucle dans le lait à $2-3 \mathrm{~mm}$ au-dessous de la surface De cette façon, la boucle calibrée était toujours retirée du lait dans des conditions permettant une bonne répétabilité de la méthode : plan de retrait perpendiculaire à la surface du lait, vitesse constante $[16,2]$. Par pesée, nous avons réglé cette vitesse de façon à ce que le poids de lait prélevé par la boucle soit de $1 \mathrm{mg}$, et nous avons constaté que, dans ces conditions, l'erreur sur le poids prélevé était inférieure à $5 \mathrm{p}$. 100, quelle que soit l'origine du lait, sa tension superficielle ou son mode d'agitation. Par contre, le poids de lait prélevé augmentait en moyenne de 0,1 mg par $10^{\circ} \mathrm{C}$ d'élévation de température entre 0 et $40^{\circ} \mathrm{C}$.

\footnotetext{
* Vortex-Genie, Scientific Industries Inc. (U.S.A.), modèle K 550-GE.
} 
b) Méthode PAR Dilutions déctmales (MDD)

Après l'agitation "standard " décrite plus haut le lait a été dilué à $1 / 1000$ en utilisant à chacune des trois dilutions décimales, des tubes contenant exactement $9 \mathrm{ml}$ de solution de Ringer dilué à $1 / 4$ et des pipettes de $1 \pm 0,01 \mathrm{ml}$. Ces tubes étaient agités 5 à $10 \mathrm{~s}$ à l'aide du Vortex. Sur chaque échantillon de lait on a répété trois fois cette dilution et on a inoculé 2 boîtes de Petri à partir de chaque dilution à $1 / 1000$ (soit au total 6 boîtes par échantillon de lait).

\section{Etudes complémentaires}

Afin de faciliter la comparaison des résultats des dénombrements effectués avec les deux méthodes précédemment décrites, nous avons effectué les études complémentaires suivantes :

a) DÉTERmination, PAR EXAMEN MiCrosCopique, DE L'EFFICACITÉ DE TROIS MODES D'AGITATION DU LAIT CRU

L'efficacité de l'agitation standard (AS), du traitement pendant 5 à 15 min à température ambiante par les ultrasons (US) et de l'agitation par turbine (UT) pendant $30 \mathrm{~s}$ à $2 \mathrm{~min}$ sur le lait a été évaluée par estimation à l'aide du microscope, du nombre approximatif de cellules présentes dans les amas microbiens [3]. Pour cette étude nous avons utilisé huit échantillons de lait cru d'origines diverses, conservés $5 \mathrm{~h}$ à $37^{\circ} \mathrm{C}$ ou $18 \mathrm{~h}$ à $20^{\circ} \mathrm{C}$ de façon à avoir des nombres de micro-organismes suffisamment élevés (dépassant $1 \mathrm{million} / \mathrm{ml}$ ) pour permettre l'examen d'un nombre appréciable d'amas microbiens sur une préparation microscopique.

Cet examen microscopique a été réalisé tout de suite après l'agitation du lait et renouvelé après $5 \mathrm{j}$ de conservation du lait ainsi agité à $0^{\circ} \mathrm{C}$.

b) COMPARAISON, DANS DES CONDITIONS OPTIMALES, DES DEUX MÉTHODES DE DÉNOMBREMENT

Afin, d'une part, de vérifier par numérations microbiennes la précision de notre dispositif mécanique de prélèvement à l'anse calibrée et d'autre part de déterminer la répétabilité de la MST et de la MDD lorsqu'elles sont appliquées à un liquide plus homogène que le lait, nous avons utilisé ces deux méthodes pour le dénombrement de micro-organismes en suspension dans du bouillon nutritif dont la tension superficielle était comparable à celle du lait entier (environ 40 dynes $/ \mathrm{cm}$ ). Ces suspensions étaient préparées à partir de micro-organismes isolés du lait cru (deux microcoques, un streptocoque, deux bacilles à Gram négatif et deux souches de corynébactéries); une colonie de chaque type de micro-organismes a été dispersée dans le bouillon par traitement UT pendant $30 \mathrm{~s}$. On préparait ensuite des dilutions de ces suspensions dans le même bouillon de façon à ce que l'on ait de $3 \times 10^{4}$ à $6 \times 10^{5}$ cellules $/ \mathrm{ml}$. 
Les numérations par la MDD ont été faites en quintuple, et celles par la MST en triple

c) INFLUENCE SUR LE DÉNOMBREMENT DE DIVERS MODES D'AGITATION DES DILUTIONS DE LAIT CRU

Afin de déterminer l'influence éventuelle sur les résultats de dénombrement suivant la MDD des pipetages effectués à chaque dilution et de l'agitation des tubes de dilution, on a préparé des dilutions directes de lait à $1 / 1000$ en ajoutant environ $0,1 \mathrm{ml}$ de lait agité de façon «standard » à $100 \mathrm{ml}$ de solution de Ringer stérile diluée au quart. Le mélange était assuré par cinq retournements du flacon, puis, sur des parts aliquotes, l'un des traitements suivants a été effectué :

$\mathrm{T}_{1}$ et $\mathrm{T}_{2}$ : aucune agitation (témoins).

P : $\quad 10$ pipetages successifs.

AS : agitation « standard ».

V45: $\quad 45 \mathrm{~s}$ d'agitation avec le vibrateur Vortex réglé au maximum.

Les traitements suivants ont été ajoutés à cette étude :

US $5 \mathrm{~min}$ : $5 \mathrm{~min}$ d'agitation dans la cuve à ultrasons à température ambiante.

UT $30 \mathrm{~s}$ : $30 \mathrm{~s}$ d'agitation avec la turbine,

de façon à comparer l'efficacité de ces traitements appliqués d'une part à une dilution de lait à $1 / 1000$ et d'autre part au lait entier.

L'inoculation des boîtes était faite en triple à l'aide de pipettes de $1 \mathrm{ml}$ avec une erreur sur le volume inférieure à $1 \mathrm{p} .100$. Le témoin $\mathrm{T}_{1}$ était inoculé en premier et $\mathrm{T}_{2}$ en fin d'opération (au maximum 10 min plus tard) pour mettre en évidence une éventuelle multiplication microbienne au cours des manipulations [15].

\section{Conditions de culture}

Tous les dénombrements ont été effectués sur milieu Plate Count Agar (Difco) avec incubation des boîtes de Petri 3 jours à $30^{\circ} \mathrm{C}$. En outre, dans l'étude précédente $(2, c)$ nous avons effectué un ensemencement en simple sur milieu Mannitol Salt Agar (Difco), sur milieu de Barnes [5] et sur Nutrient Agar (Difco) additionné de $2 \mathrm{mg} / \mathrm{l}$ de cristal violet de façon à estimer respectivement les nombres de microcoques, de streptocoques et de bactéries à Gram négatif.

\section{Exploitation des résultats}

Les résultats des dénombrements des colonies sur les boîtes de Petri ont été soumis à une analyse de variance [1] après transformation logarithmique des données. Les écarts entre les moyennes ont été examinés en prenant comme critère la plus petite différence significative [23] et comme référence, soit la MDD soit la MST, l'une et l'autre appliquées au lait agité de façon standard. 


\section{RESULTATS}

\section{Examen direct de l'efficacité de l'agitation du lait}

Le tableau 1 montre l'influence de trois modes d'agitation du lait cru sur la taille des amas formé par trois types de bactéries. On observe que l'agitation avec la turbine disperse à peu près complètement les amas microbiens; le traitement pendant 5 min aux ultrasons est plus efficace que l'agitation standard mais beaucoup moins que celle utilisant la turbine. L'augmentation de la durée, au-delà de $5 \mathrm{~min}$, du traitement aux ultrasons provoquait la formation de grains de beurre; l'étude du facteur " durée " n'a donc pas été poursuivie. La prolongation de la durée d'agitation (jusqu'à $2 \mathrm{~min}$ ) avec la turbine conduisait également à la formation de beurre et n'entraînait pas la dispersion des amas restants.

Nous n'avons pas noté d'agglutination des micro-organismes après conservation pendant 5 jours à $0^{\circ} \mathrm{C}$ des échantillons traités par les différentes méthodes.

\section{Comparaison, dans des conditions optimales, des deux méthodes de dénombrement}

Les résultats des dénombrements microbiens effectués sur des suspensions microbiennes dans un bouillon nutritif (milieu homogèné) par la MST et par la MDD ont été soumis à l'analyse statistique suivante : comparaison de moyennes, corrélation et régression linéaire, après transformation logarithmique de base 10 .

Nous avons obtenu, entre les nombres de colonies donnés par la MDD et par la MST un coefficient de corrélation de 0,986 et l'équation de régression :

$$
\log _{10} \mathrm{MDD}=0,98 \log _{10} \mathrm{MST}+0,095 .
$$

Les deux méthodes de dénombrement donnent donc des résultats qui ne diffèrent donc pas significativement (pente de la droite peu différente de 1 , origine peu différente de 0 ).

Sur le plan de la répétabilité, la MST s'est révélée être un peu moins bonne que la MDD (variance respectivement de 0,0043 et de 0,0028 ). Nous avons également constaté que cette variance augmentait considérablement lorsque le nombre de colonies diminuait. Ainsi, avec la MDD, la variance était de 0,0009 pour 100 à 600 colonies par boîte, et de 0,0062 pour 30 à 100 colonies par boîte; avec la MST, les variances correspondantes étaient de 0,0029 et de 0,0068.

\section{Influence de divers modes d'agitation de la dilution de lait sur les résultats des dénombrements microbiens}

Les résultats de cette étude sont présentés dans le tableau 2. Les pipetages n'ayant manifestement aucune influence sur la disper- 
TABLEAU 1

Influence de trois modes d'agitation du lait sur la taille des amas microbiens

\begin{tabular}{|c|c|c|c|c|c|c|c|c|}
\hline \multirow{2}{*}{$\begin{array}{c}\text { Types de } \\
\text { micro-organismes }\end{array}$} & \multirow{2}{*}{$\begin{array}{c}\text { Mode } \\
\text { d'agitation }\end{array}$} & \multirow{2}{*}{$\begin{array}{l}\text { Nombre } \\
\text { d'amas } \\
\text { examinés }\end{array}$} & \multicolumn{6}{|c|}{ p. 100 moyens d'amas contenant* } \\
\hline & & & $\begin{array}{c}1 \text { à } 2 \\
\text { cellules }\end{array}$ & $\begin{array}{l}3 \text { à } 6 \\
\text { cellules }\end{array}$ & $\begin{array}{c}7 \text { à } 10 \\
\text { cellules }\end{array}$ & $\begin{array}{l}11 \text { à } 20 \\
\text { cellules }\end{array}$ & $\begin{array}{l}21 \text { à } 50 \\
\text { cellules }\end{array}$ & cellules \\
\hline Streptocoques & $\begin{array}{l}\text { AS } \\
\text { US, } 5 \text { min } \\
\text { UT }\end{array}$ & $\begin{array}{l}318 \\
354 \\
405\end{array}$ & $\begin{array}{l}36 \\
53 \\
77\end{array}$ & $\begin{array}{l}12 \\
24 \\
20\end{array}$ & $\begin{array}{r}13 \\
10 \\
2\end{array}$ & $\begin{array}{r}17 \\
3 \\
<1\end{array}$ & $\begin{array}{r}12 \\
6 \\
<1\end{array}$ & $\begin{array}{r}10 \\
4 \\
0\end{array}$ \\
\hline Microcoques & $\begin{array}{l}\text { AS } \\
\text { US, } 5 \text { min } \\
\text { UT }\end{array}$ & $\begin{array}{r}113 \\
84 \\
129\end{array}$ & $\begin{array}{l}30 \\
59 \\
74\end{array}$ & $\begin{array}{l}21 \\
13 \\
20\end{array}$ & $\begin{array}{r}14 \\
12 \\
3\end{array}$ & $\begin{array}{r}23 \\
9 \\
2\end{array}$ & $\begin{array}{r}10 \\
7 \\
1\end{array}$ & $\begin{array}{l}2 \\
0 \\
0\end{array}$ \\
\hline Bâtonnets & $\begin{array}{l}\text { AS } \\
\text { US, } 5 \text { min } \\
\text { UT }\end{array}$ & $\begin{array}{l}410 \\
389 \\
384\end{array}$ & $\begin{array}{l}67 \\
86 \\
94\end{array}$ & $\begin{array}{r}16 \\
10 \\
6\end{array}$ & $\begin{array}{l}8 \\
2 \\
0\end{array}$ & $\begin{array}{l}7 \\
1 \\
0\end{array}$ & $\begin{array}{l}2 \\
1 \\
0\end{array}$ & $\begin{array}{l}0 \\
0 \\
0\end{array}$ \\
\hline
\end{tabular}

AS : agitation "standard».

US, $5 \mathrm{~min}$ : $5 \mathrm{~min}$ de traitement aux ultrasons à $10-20^{\circ} \mathrm{C}$.

UT : $30 \mathrm{~s}$ de traitement à l'Ultra-Turrax.

* Moyenne arithmétique des résultats obtenus avec huit échantillons de lait. 


\section{TABLEAU 2}

Influence de l'agitation des dilutions à 1/1000 de lait cru sur les résultats de dénombrement de la flore totale

\begin{tabular}{l|c|c|c}
\hline $\begin{array}{c}\text { Méthode } \\
\text { d'agitation }\end{array}$ & Etendue & $\begin{array}{c}\text { Nombre de } \\
\text { colonies/boîte (a) }\end{array}$ & $\begin{array}{c}\text { Répétabilité } \\
\text { (c) }\end{array}$ \\
\cline { 1 - 2 } Témoin 1 (initial) & $20-463$ & 95 & (b) \\
Témoin 2 (après 10 min) & $28-470$ & 96 & 0,0039 \\
Vortex (45 s) & $23-481$ & 99 & 0,0041 \\
Agitation standard & $22-554$ & 102 & 0,0049 \\
Ultrasons (5 min) & $27-876$ & 112 & 0,0037 \\
Turbine (30 s) & $35-1021$ & 176 & 0,0049 \\
& & & 0,0029 \\
\hline
\end{tabular}

(a) Moyenne logarithmique de 26 échantillons analysés en triple.

(b) Les moyennes réunies par une accolade ne diffèrent pas significativement entre elles au seuil $\mathrm{P}=0,05$.

(c) Variance calculée à partir du $\log _{10}$ du nombre de colonies par boîte de Petri.

sion des amas microbiens, nous en avons abandonné l'étude après l'examen d'une dizaine d'échantillons.

L'analyse de variance faisait apparaître un terme interaction " Echantillon $\times$ Mode d'agitation » hautement significatif (carré moyen égal à 0,04$)$.

Les dénombrements sur milieux sélectifs n'ont pas permis de révéler si un groupe déterminé de micro-organismes était responsable de l'élévation du nombre de colonies après traitement US ou UT : le nombre de colonies sur le milieu de Barnes ou sur Mannitol Sált Agar restait très faible (généralement moins de 30 colonies) même après agitation avec la turbine. Sur Nutrient Agar additionné de $2 \mathrm{mg} / \mathrm{l}$ de cristal violet, le nombre de colonies était plus élevé mais sans rapport avec l'augmentation du nombre de colonies constatée sur PCA.

\section{Influence du mode d'agitation du lait cru sur la précision et la répétabilité de la méthode simplifiée de Thompson (MST)}

Les résultats moyens de dénombrements microbiens effectués sur le lait cru par la MST sont présentés dans le tableau 3. La MST, avec 
Influence de divers modes d'agitation du lait sur les résultats de dénombrement de la flore totale suivant la méthode simplifiée de Thompson (MST)

Nombre moyen de colonies par boîte (a)

\begin{tabular}{|c|c|c|c|c|c|c|c|}
\hline \multirow{3}{*}{ Expérience } & \multirow{3}{*}{$\mathrm{N}(\mathrm{b})$} & \multirow{3}{*}{ MDD (c) } & \multicolumn{5}{|c|}{ Méthode simplifiée de Thompson (MST) } \\
\hline & & & Agitation & Vortex & Ultrasons & $(80 \mathrm{kHz})$ & \multirow{2}{*}{$\begin{array}{l}\text { Turbine } 30 \mathrm{~s} \\
\text { à } 20000 \mathrm{t} / \mathrm{min}\end{array}$} \\
\hline & & & & & $5 \mathrm{~min}$ & $15 \mathrm{~min}$ & \\
\hline 1 & 5 & $\begin{array}{c}186 \\
(0,0049)\end{array}$ & $\begin{array}{c}182 \\
(0,0030)\end{array}$ & $\begin{array}{c}194 \\
(0,0049)\end{array}$ & $\begin{array}{c}\square \\
229 * \\
(0,0028)\end{array}$ & $\begin{array}{c}\square \\
269 * \\
(0,0033)\end{array}$ & - \\
\hline 2 & 10 & $\begin{array}{c}48 \\
(0,0106)\end{array}$ & $\begin{array}{c}66^{*} \\
(0,0178)\end{array}$ & $\begin{array}{c}46 \\
(0,0035)\end{array}$ & - & $\begin{array}{c}\square \\
89^{*} \\
(0,0053)\end{array}$ & - \\
\hline 3 & 15 & $\begin{array}{c}44 \\
(0,0017)\end{array}$ & $\begin{array}{c}43 \\
(0,0016)\end{array}$ & $\begin{array}{c}\square \\
34^{*} \\
(0,0053)\end{array}$ & $\begin{array}{c}\square \\
50 * \\
(0,0044)\end{array}$ & - & - \\
\hline 4 & 37 & $\begin{array}{c}58 \\
(0,0064)\end{array}$ & $\begin{array}{c}48^{*} \\
(0,0113)\end{array}$ & $\begin{array}{c}63 \square \\
(0,0057)\end{array}$ & $\begin{array}{c}\square \\
81 * \\
(0,0052)\end{array}$ & - & - \\
\hline 1 à 4 & 67 & $\begin{array}{c}58 \\
(0,0054)\end{array}$ & $\begin{array}{c}54 \\
(0,0084)\end{array}$ & $\begin{array}{c}57 \\
(0,0048)\end{array}$ & $\begin{array}{c}\square \\
77^{\star} \\
(0,0043)\end{array}$ & - & - \\
\hline 5 & 25 & $\begin{array}{c}60 \\
(0,0057)\end{array}$ & $\begin{array}{c}63 \\
(0,0061)\end{array}$ & - & - & - & $\begin{array}{l}\quad \square \\
95^{*} \\
(0,0046)\end{array}$ \\
\hline
\end{tabular}

(a) Moyennes logarithmiques des nombres de colonies par boîtes de Petri avec, entre parenthèses, l'estimation de la variance de répétabilité des numérations calculée à partir des logarithmiques.

(b) $\mathrm{N}$ : nombre d'échantillons.

(c) MDD : méthode par dilutions décimales avec agitation "standard " du lait.

Les moyennes marquées * ou $\square$ diffèrent significativement au seuil $P=0,05$ respectivement de la méthode MDD ou de la méthode MST aver aoitation "standard " 
agitation standard, donne des résultats qui s'écartent significativement de la MDD avec agitation standard prise comme référence dans deux expériences sur cinq, mais sur l'ensemble des résultats des cinq expériences (92 échantillons), les deux méthodes ne diffèrent pas significativement; on remarquera que dans les deux expériences où elles donnent des résultats significativement différents (exp. 2 et 4), leur répétabilité est également moins bonne que dans les autres expériences (variances comprises entre 0,0064 et 0,0178).

L'agitation supplémentaire du lait pendant 2 min par le Vortex donne, sur l'ensemble des expériences, des résultats qui ne s'écartent pas significativement de la MDD ou de la MST appliquées au lait agité de façon «standard », tant sur le plan de la moyenne que sur celui de la répétabilité.

Nous avons constaté qu'il fallait au moins 5 min de traitement du lait aux ultrasons (à température ambiante) pour obtenir une augmentation significative du nombre de colonies par rapport à l'agitation "standard ". Une prolongation du traitement après $15 \mathrm{~min}$ ne permettait pas d'accroître ce nombre ; nous avons constaté de même qu'une augmentation de température de 0 à $25^{\circ} \mathrm{C}$ était sans effet ; par contre, un traitement de 5 min à $40^{\circ} \mathrm{C}$ permettait d'obtenir une augmentation significative du nombre de colonies pouvant être attribuée aussi bien à une dispersion accrue des amas microbiens qu'à l'accroissement du volume de lait prélevé à l'anse. Pour l'ensemble des expériences 1 à 4 (57 échantillons) un traitement de $5 \mathrm{~min}$ aux ultrasons, à température ambiante, permet d'augmenter la moyenne du nombre de colonies de 0,1 unité log par rapport à l'agitation standard, ce qui correspond à 1,25 fois la moyenne obtenue avec cette agitation.

Enfin, l'agitation par turbine (exp. 5), permet d'accroître significativement le nombre de colonies par rapport à l'agitation standard : l'augmentation moyenne obtenue est de 0,2 unité $\log$, soit environ 1,6 fois la moyenne log obtenue avec l'agitation standard. C'est un résultat un peu supérieur à celui obtenu par Wanser et Hartman [30] pour le lait, mais plus faible que celui que nous avons obtenu en traitant les dilutions de lait (tab. 2).

Dans l'ensemble, la répétabilité de la MST paraît moins bonne lorsqu'elle est appliquée au lait agité seulement de façon standard que lorsqu'elle est appliquée au lait agité de façon plus énergique. Cette répétabilité ne diffère cependant pas significativement de celle de la méthode classique appliquée au lait agité uniquement de façon standard (test d'homogénéité des variances de Bartlett non significatif [23]). Par ailleurs, comme dans l'étude sur les dilutions de lait, l'analyse de variance faisait apparaître un terme d'interaction "Echantillon $\times$ Mode d'agitation » très significatif (de 0,015 à 0,119 ) dans toutes les expériences. Cela signifie que l'effet de l'agitation varie suivant l'origine des échantillons, donc probablement suivant la 
nature de la flore microbienne du lait, et/ou suivant la nature même du lait.

\section{DISCUSSION}

Notre étude montre que les deux méthodes de dénombrement de la flore totale appliquées à du lait cru conservé, au plus $48 \mathrm{~h}$ à moins de $5^{\circ} \mathrm{C}$, donnent en moyenne, des résultats qui ne diffèrent pas significativement, à condition que pour chacune d'elles, le lait soit agité de la même façon. Ces résultats confirment ceux d'autres auteurs $[29,18,22,20,11]$.

Il est évident que si les dilutions de lait sont elles aussi agitées énergiquement ou conservées plus de $10 \mathrm{~min}$, on peut observer une différence significative entre les deux méthodes de dénombrement $[14,15]$. Le diluant (eau physiologique ou solution de Ringer) utilisé pour les dilutions peut également avoir un effet nocif sur les bactéries $[26,13,6]$, et introduire de ce fait, une différence entre les deux méthodes de dénombrement; il en est de même d'une erreur sur le volume de diluant, erreur qui peut atteindre une valeur de $17 \mathrm{p} .100$ d'après Jennisson et Wadsworth [17]. Pour toutes ces raisons, la méthode de dénombrement simplifiée mécanisée paraît être, dans le principe, meilleure que celle faisant intervenir des dilutions et pourrait remplacer cette dernière, comme le suggèrent Bradshaw et al. [8] ; cependant, nous avons observé, confirmant en cela les résultats d'une précédente étude [22], qu'elle a, bien que mécanisée, une répétabilité un peu moins bonne que la méthode par dilutions. Cela n'est pas explicable par une variation du volume de lait inoculé, car la vitesse de retrait de la boucle calibrée était relativement constante. On peut donc raisonnablement penser que la partie superficielle du lait (ou des suspensions microbiennes) dans laquelle plonge la boucle n'est pas homogène, en dépit d'une agitation énergique.

Une agitation du lait plus énergique que l'agitation "standard » permet d'obtenir des nombres plus élevés de colonies. Cependant cette augmentation n'est pas aussi importante que le laissaient prévoir les résultats de l'examen microscopique ; elle est au plus de 60 p. 100 avec le mode d'agitation dispersant presque complètement les amas microbiens. Cela peut s'expliquer par le fait que la majorité des bactéries du lait cru conservé au plus $48 \mathrm{~h}$ à moins de $5^{\circ} \mathrm{C}$ ne sont pas sous forme d'amas importants, probablement parce que ces amas sont désagrégés au cours de la traite, du fait de leur écrasement dans les raccords de machine à traire (qui sont les principales sources de pollution du lait) et de leur éclatement au cours du pompage du lait. On n'assiste pas non plus, en $48 \mathrm{~h}$, à une multiplication significative de la flore psychrotrophe du lait qui pourrait provoquer la formation d'agrégats microbiens [28], ni à une agrégation des micro-organismes que le lait contient. 
Par ailleurs, une agitation énergique du lait n'a pas d'influence sur la répétabilité des deux méthodes de dénombrement. Les valeurs plus faibles de la variance de répétabilité de l'agitation par turbine sont le fait seulement de l'augmentation du nombre de colonies. En effet, nous avons mentionné à plusieurs reprises la relation existant entre la variance de répétabilité des dénombrements (calculée après conversion $\log _{10}$ ) et le nombre de colonies sur les boîtes de Petri. Cette relation est due au fait que les nombres de colonies sur les boîtes de Petri suivent approximativement une loi de Poisson [25]. La conversion $\log _{10}$ des données conserve cette relation; il en résulte théoriquement que la variance de répétabilité diminue lorsque le nombre de colonies augmente. C'est ce que nous avons effectivement observé. L'absence de transformation, ou la transformation $\sqrt{\mathrm{x}}$, n'élimine pas cette relation.

En conclusion, nos résultats confirment que la méthode simplifiée de dénombrement de la flore totale donne, pour un mode d'agitation du lait donné, des résultats similaires à ceux d'une méthode par dilutions parfaitement réalisée (erreur négligeable sur les volumes, pas d'influence néfaste du diluant) ; sa moins bonne répétabilité n'affecte pas significativement sa précision et encore moins le classement des échantillons en vue du paiement du lait suivant sa qualité bactériologique.

\section{Remerciements}

Je remercie MM. Corre et Molle pour leur assistance technique et MM. Accolas, Atıclair, Bergère et Hermier pour leur aide dans la préparation du manuscrit.

\section{Rés umé}

On a étudié l'influence de quatre modes d'agitation sur les résultats de dénombrement de la flore totale du lait cru : agitation manuelle, prise comme référence, agitation manuelle suivie d'une agitation par vibrations mécaniques, agitation par ultrasons $(80 \mathrm{kHz})$ ou par turbine $(20000 \mathrm{t} / \mathrm{min})$ avec, pour chaque mode d'agitation, diverses conditions de traitement.

Le traitement du lait pendant $30 \mathrm{~s}$ avec la turbine permet d'obtenir une dispersion pratiquement complète des cellules bactériennes et les nombres de colonies les plus élevés : 60 p. 100 en moyenne de plus qu'avec l'agitation manuelle. L'agitation par vibrations pendant $2 \mathrm{~min}$ du lait soumis préalablement à une agitation manuelle n'entraîne pas d'augmentation du nombre de colonies obtenues. Au moins 5 min de traitement aux ultrasons à température ambiante sont nécessaires pour avoir une dispersion importante des amas microbiens et une augmentation significative du nombre de colonies 
(en moyenne 25 p. 100 de plus qu'avec l'agitation manuelle). La prolongation de la durée de ce traitement occasionne la formation de grains de beurre sans disperser les amas microbiens restants.

Pour un même mode d'agitation du lait (agitation manuelle), la méthode de dénombrement simplifiée à l'aide d'une anse calibrée donne des nombres de colonies qui ne diffèrent pas significativement, en moyenne, de ceux obtenus avec la méthode de dénombrement par dilutions décimales. Les modes d'agitation étudiés n'ont pas d'influence significative sur la répétabilité de ces méthodes de dénombrement.

\section{S u m m a r y}

EFFECT OF SEVERAL SHAKING METHODS ON PLATE COUNTS

OF RAW-MILK BACTERIA

The effect of four shakings on the bacterial counts of raw milk was studied: "standard" shaking (25 complete up-and-down movements of about $30 \mathrm{~cm}$ in $5-10 \mathrm{~s}$ ), standard shaking followed by mechanical vibrations (Vortex-Genie, Scientif Industries Inc .USA model K 550.GE, max. speed), ultrasonic treatment $(80 \mathrm{kHz})$, or by a mixer (Ultra-Turrax, Janke \& Kunkel KG, West Germany, model TP 18-10, 20,000 rev/min), the treatment conditions beeing different for each shaking procedure.

Treatment of the milk for $30 \mathrm{~s}$ with the mixer resulted in an altmost complete bacterial clumps dispersion and to the highest bacterial counts (on an average 60 p. 100 more than with the standard shaking). Mechanical vibrations for 2 min of milk previously shaken by the standard technique did not result in any increase in the numbers of colonies. An ultrasonic treatment of at least $5 \mathrm{~min}$ at room temperature was required for obtaining a marked dispersion of the bacterial clumps and a significant increase in the number of colonies (on an average 20 p. 100 more than with the standard shaking). A lengthening of this treatment resulted in butter formation without dispersing the residual bacterial clumps.

The plate loop method and the standard plate method of bacterial enumeration gave similar results provided that the same milk shaking technique was used. The repeatability of these methods was not significantly affected by the shaking methods studied.

Reçu pour publication en février 1980.

\section{Bibliographie}

[1] AFNOR (1970). - Fidélité d'une méthode d'essai. Guide pratique pour les essais interlaboratoires. Norme NF X06-041. 
[2] Anonyme (1967). - Studiegroep kwaliteitsbepaling melk van de F.N.Z. De bepaling van het kiemgetal van melk et behulp van een öse. Officieel Org. Ned. Zuivelbard, 59, 763-766.

[3] APHA (American Public Health Association) (1972). - Standard Methods for the examination of dairy products. 13e Ed. USPHS, Washington, D.C.

[4] Auclair (J.) et Portman (A.) (1959). - Action inhibitrice des bactéries sur les streptocoques lactiques des levains de fromagerie. $X V^{\mathrm{e}}$ Congrès International de Laiterie, 2, 580-586.

[5] BARNeS (E. M.) (1956). - Methods for the isolation of faecal streptococci (Lancefield Group D) from bacon factories. Journal of Applied Bacterio$\log y, 19,193-203$.

[6] Berry (J. M.), Mc Neill (D. A.) and Witter (L. D.) (1969). - Effect of delays in pour plating on bacterial counts. Journal of Dairy Sciences, 52, 1456-1457.

[7] Bottazzi (V.), Dellaglio (F.) and SarRa (P. G.) (1972). - Creaming of milk and bacterial agglutination of microorganisms. IV. Mechanism causing fat and bacterial agglutination. Scienza e Tecnica Lattiero-Casearia, 23, 287-304.

[8] Bradshaw (J. G.), Francis (D. W.), Peeler (J. T.), Leslie (J. E.), Twedt (R. M) and READ (R. B. Jr.) (1973). - Mechanical preparation of pour plates for viable bacteria counts of milk samples. Journal of Dairy Sciences, 56, 1011-1016.

[9] Donnelly (C. B.) Peeler (J. T.) and Black (L. A.) (1966). - Evaluation of state central milk laboratories by statistical analyses of standard plate counts. Journal of Milk and Food Technology, 29, 19-24.

[10] Kanno (C.), Emmons (D. B.), Elliott (J. A.) and Beckett (D. C.) (1966). Lactic-streptococcal agglutinins in milk and blood. XVII ${ }^{\circ}$ Congrès International de Laiterie, D 2, 499-508.

[11] Flemming (M. G.) and O'Connor (F.) (1975), - An automated loop method for determining the total count of bacteria in milk. Irish Journal of Agricultural Research, 14, 27-32.

[12 a] GRAPPIN (R.) (1975). - Mise au point sur les appareils automatiques utilisés pour la numération des germes totaux du lait : préparation des boîtes de Petri et comptage des colonies. Revue Laitière Française, 335, 629-641.

[12 b] Grappin (R.) (1976). - Guide pour l'évaluation des méthodes d'analyse de routine. Le Lait, 559-560, 608-621.

[13] Gyllenberg (H. G.) and EKLund (E.) (1959). - Selection of suitable diluents to avoid destruction of bacteria during the dilution of milk samples for plating. $X V^{\mathrm{e}}$ Congrès International de Laiterie, 3, 1331-1333.

[14] Huhtanen (C. N.), Brazis (A. R.), Arledge (W. L.), Cook (E. W.), Donnelly (C. B.), Ginn (R. E.), Murphy (J. N.), Randolph (H. E.), Sing (E. L.) and Thомpson (D. I.) (1970). - Effect of dilution bottle mixing methods on plate counts of raw milk bacteria. Journal of Milk and Food Technology, $33,(7), 269-273$.

[15] Huhtanen (C. N.), Brazis (A. R.), Arledge (W. L.), Cook (E. W.), Donnelly (C. B.), Ginn (R. E.), Jezeski (J. J.), Pusch (D.), Randolph (H. E.) and Sing (E. L.) (1972) . - Effects of time of holding dilutions on counts of bacteria from raw milk. Journal of Milk and Food Technology, 35, 126-130.

[16] JASPeR (D. E.) and Dellinger (J.) (1966). - Variations in volume of milk delivered by a standard $0.01 \mathrm{ml}$ loop. Journal of Milk and Food Technology, $29,199-200$.

[17] Jennisson (M. W.) and Wadsworth (G. P.) (1940). - Evaluation of the errors involved in estimating bacterial numbers by the plating method. Journal of Bacteriology, 39, 389-397.

[18] LeEsment (H. K.) (1964). - Undersökning av tva enkla plattspridningsmetoder för bestämning av bakterieantalet i kyllagrad leverantörmjölk. Svenska Mejeritidn., 56, 143-149. 
[19] Leesment (H. K.) und Nickels (Ch.) (1966). - Das Eichen der Burri-Ose für die Bestimmung der keimzahl nach dem Plattenverfahren. XVII ${ }^{\mathrm{e}}$ Congrès International de Laiterie, Munich B, 481-483.

[20] ORR (M. J.), Baines (S. G.) and Mc CRAE (M. C.) (1971). - The plate loop method for determing bacterial numbers in bulk-cooled milk. Milk Industry, 68, 17-22.

[21] RandolPh (H. E.) and Gould (I. A.) (1968). - Characterization of the natural inhibitors in skimmilk affecting lactic acid bacteria. Journal of Dairy Science, 51, 8-15.

[22] Richard (J.) et Auclair (J.) (1968). - Méthode simplifiée de dénombrement des germes totaux du lait. Revue Laitière française, 261, 15-18.

[23] SNedeCor (G. W.) and Cochran (W. G.) (1967). - Statistical methods. The Iowa State University Press, Ames, Iowa, U.S.A., 6th ed.

[24] Stadhouders (J.) and Hup (G.) (1970). - Complexity and specificity of euglobulin in relation to inhibition of bacteria and to cream rising. Netherland Milk Dairy Journal, 24, 79-95.

[25] Stearman (R. L.) (1955). - Statistical concepts in microbiology. Bacteriological Reviews, 19, 160-215.

[26] Straka (R. P.) and Stockes (J. L.) (1957). - Rapid destruction of bacteria in commonly used diluents and its elimination. Applied Microbiology, 5, 21-25.

[27] Tatini (S. R.), Dabbah (R.) and Olson (J. C. Jr.) (1967). - Comparison of plate loop and agar plate methods for bacteriological examination of manufacturing grade raw milk. Journal of Milk and Food Technology, 30, 112-115.

[28] The Whaiti (I. E.) and Fryer (T. F.) (1977). - The enumeration of bacteria in refrigerated milk. New Zealand Journal of Dairy Science and Techno$\log y, 12,51-57$.

[29] Thompson (D. I.), Donnelly (C. B.) and Black (L. A.) (1960). - A plate loop method for determining viable counts of raw milk. Journal of Milk and Food Technology, 23, 167-171.

[30] Wanser (B. E.) and Hartman (P. A.) (1962). - Agitation of milk samples for colony counts of raw milk. Journal of Milk and Food Technology, 25, 5-6.

[31] Wright (E. O.), Reinbold (G. W.), Burmeister (L.) and Mellon (J.) (1970). Prediction of standard plate count of manufacturing-grade raw milk from the plate loop count. Journal of Milk and Food Technology, 33, 168-170.

[32] Wright (E. O.), Reinbold (G. W.), Burmeister (L.) and Mellon (J.) (1972). Prediction of standard plate count of manufacturing-grade raw milk from the plate loop count. Journal of Milk and Food Technology, 35, 141. 